\title{
The (continuing) impact of Covid-19: an editorial
}

\author{
Thomas Guenther $^{1,2} \cdot$ Frank Verbeeten $^{1}$ (D)
}

Accepted: 13 September 2021 / Published online: 19 October 2021

(c) The Author(s), under exclusive licence to Springer-Verlag GmbH Germany, part of Springer Nature 2021

At the start of the new academic year, we can reflect upon a 'full Covid academic year', i.e., an academic year'20-'21 that has been fully affected by Covid-19. At the start of the pandemic, there was the idea that Covid-19 would have a short impact: teaching was going digital (with all issues relating to exams), yet the idea was that would not last long. During the Spring semester, many institutions started to realize that the pandemic would have a large impact upon the upcoming academic year, which required digital teaching and examinations on a more structural basis. This required different didactical approaches, adoptions of exam regulations as well as new technical skills from professors and lecturers, and a more organized approach to online course delivery. Now, we have had students in a 1-year master program that may have never met any professor in the program in person. And students that have started their bachelor program 'at the wrong time' (i.e., September 2019) have had more classes online than onsite.

In addition to teaching, also research has been affected and gone online (at least in many Western countries). Qualitative researchers have difficulties obtaining data through physical interviews; on the other hand, the online communications tools (Zoom, Teams) provide opportunities to interview people across the world. Experimental researchers cannot obtain their data from physical experimental labs, yet several of them have moved to online environments (including Amazon's MTurk or Prolific Academic). Conferences have gone online due to uncertainties on travel restrictions worldwide and, as a consequence of that, the number of attendants. Hotel facilities may be difficult to obtain, even though researchers are willing to spend their research budgets (at least, that is what a colleague indicated). While many of us are hoping that we can speak to each other in person soon (both in the office as well as in an onsite conference), there are still many uncertainties in the near future. Even though many people that want to get a 'jab' are already vaccinated, the

Frank Verbeeten

f.h.m.verbeeten@uva.nl

Thomas Guenther

thomas.guenther@tu-dresden.de

1 Amsterdam, Netherlands

2 Technische Universitaet Dresden, Dresden, Germany 
Covid-19 pandemic is likely to affect our personal as well as academic lives in the (near) future.

As a response to the Covid-19 pandemic, the Journal of Management Control has adjusted its review processes to provide more flexibility to reviewers. Given that many of us have to work from home, reviewing may not always be possible due to homeschooling, care for parents or other social demands that reviewers face. In addition, given these pressures and demands (in the work environment as well as private life), we are well aware that research may suffer. In response to this, some institutions have adjusted their research requirements in the allocation of research funds and time (for example, by extending the period in which 'publications count'). At the Journal of Management Control, we also tend to be flexible for the return timelines for both authors as well as reviewers; we therefore encourage authors and reviewers to contact us if deadlines are unrealistic, given other demands.

Despite all these challenges, pressures and demands, this third issue of the Journal of Management Control includes four very interesting research papers. Vincent Chong, Chanel Loy, Isabel Wang and David Woodliff examine the effect of the negotiator's role (seller vs. buyer), leadership tone (supportive vs. non-supportive), and social value orientation (prosocials vs. proselfs) on expected transfer prices outcomes as expressed by negotiation managers. Using a $2 \times 2$ experiment, they find that prosocial managers' expect that transfer prices will be closer to an equal-profit price relative to proself managers. In addition, they find that the transfer price expectation gap between proself selling managers and buying managers under a non-supportive leadership tone is larger than it is under a supportive leadership tone, suggesting that the negative effect of proself as compared to prosocial managers can be mitigated by a supportive leadership tone.

The second paper, by Walid Cheff, Ahmed Abdel-Maksoud and Muhammad Omer Farooq, investigates the mediating role that integrating corporate social responsibility (CSR) into the management control systems (MCS) of small and medium-sized enterprises (SMEs) can play in the relationship between CSR initiatives and organizational performance. Using responses from 117 SMEs in an emerging economy, they find that CSR initiatives in SMEs influence organizational performance both directly and indirectly through MCS, and that management accountants play a key role in this.

Thuy-Van Tran, Sinikka Lepistö and Janne Järvinen examine the relationship between subjectivity in performance evaluations, and three dimensions of justice perceptions in an emerging economy. Results from a survey of 160 middle managers in Vietnam indicate that subjective evaluation is associated predominantly with negative effects. They find that, in an emerging economy like that of Vietnam, subjective evaluation reduces interactional justice perception, which in turn decreases the perception of procedural and distributive justice. Their mediating effects suggest that the reason subjective evaluation influences employee procedural/distributive justice perceptions lies in the interactional justice perceived from supervisors. Their research clarifies the effects of subjective evaluation on the dimensions of justice perception and contributes to the literature on performance evaluation and organizational justice in a non-Western context. 
Finally, Thomas Kristensen and Henrik Saabye provide insight in how organizational members' perceptions of the enabling use of performance measures is increased when the case company adopted lean principles in one of its productionsupport departments. The theory of enabling formalization is applied to gauge and understand the extent to which organizational members perceive performance measures, such as key performance indicators (KPIs), as enabling (i.e. as a vehicle creating continuous improvement). They find a positive relation between changing the context of performance measures and the perceived level of enabling use of KPIs.

We hope that the readers of the Journal of Management Control may draw new insights from this issue and that the authors can thereby contribute to expand our knowledge in the field of management accounting and control, both for practice and research.

Amsterdam, August 2021.

For the team of editors: Frank Verbeeten and Thomas Guenther Managing editors.

Publisher's Note Springer Nature remains neutral with regard to jurisdictional claims in published maps and institutional affiliations. 